\title{
De la adhesión voluntaria a las puertas abiertas a medias: la arbitrariedad en el cumplimiento de un principio cooperativo
}

\author{
From voluntary membership to open doors to half: \\ arbitrariness in the compliance of a cooperative principle
}

José Eduardo de Miranda

UNICESUMAR (Brasil)

Sumario: Introducción; I. Una cuestión de principio: ¿pero que es principio?; II. Los principios del Cooperativismo; III. El principio de la adhesión voluntaria: las puertas abiertas; IV. Las puertas abiertas a medias: una práctica arbitraria que impide el cumplimiento del primer principio cooperativo por sociedad cooperativa de médicos brasileña; V. A título de conclusión; VI. Referencias.

Summary: Introduction; I. A question of principle: but what is the principle? II. The principles of Cooperatives; III. The principle of voluntary membership: open doors; IV. Half-open doors: an arbitrary practice that prevents compliance with the first cooperative principle by a cooperative society of Brazilian doctors; $\mathrm{V}$. By way of conclusion; VI. References.

Resumen. Los principios del Cooperativismo proceden de la interpretación y de los estudios sobre los Estatutos Sociales y los demás reglamentos de la Sociedad Cooperativa de los Probos Pioneros de Rochdale. En este sentido, la Alianza Cooperativa Internacional se encarga de preservar la actualidad del debate sobre los principios cooperativos, como forma de salvaguardarse la esencia de la identidad del sistema cooperativo. Asimismo, algunos principios, como el principio de la adhesión libre y abierta son refutados por cooperativas que adoptan criterios personales y arbitrarios para optimizar la candidatura y aprobación del ingreso de nuevos miembros al cuadro asociativo. Es importante, por esto, un análisis del sentido de los principios cooperativos, y el alcance de la adhesión libre y voluntaria, para que se pueda confirmar que la esencia del Cooperativismo transciende el sentido económico.

Palabras clave: Principios cooperativos. Adhesión voluntaria. Arbitrariedad.

1 Doctor en Derecho. Correo electrónico: jemiranda@mirandacorrealima.com 
Abstract: The principles of Cooperativism come from the interpretation and studies on the Social By-Laws and other regulations of the Cooperative Society of Pioneering Probes of Rochdale. In this sense, the International Cooperative Alliance is in charge of preserving the topicality of the debate on cooperative principles, as a way of safeguarding the essence of the identity of the cooperative system. Also, some principles, such as the principle of free and open membership, are refuted by cooperatives that adopt personal and arbitrary criteria to optimize the candidacy and approval of new members to join the membership. It is important, therefore, an analysis of the meaning of cooperative principles, and the scope of free and voluntary adherence, so that it can be confirmed that the essence of Cooperativism transcends economic sense.

Keywords: Cooperative principles. Voluntary membership. Arbitrariness. 
Princípio é a estruturação de um sistema de ideias, pensamentos ou normas por uma ideia mestra, por um pensamento chave, por uma baliza normativa, donde toda as demais ideias, pensamentos ou normas derivam, se reconduzem elou subordinam.

(Espíndola, 2002, 152)

\section{Introducción}

Formateado bajo la influencia de una disposición moral que surgió en los estatutos de la Cooperativa de los Probos Pioneros de Rochdale, el Cooperativismo se ocupó de conformar las características esenciales de las sociedades cooperativas, indispensables tanto para satisfacer las necesidades de sus miembros, como para instituir un orden económico más justo, mediante la creación de mejores comunidades y sociedades más humanas.

En este sentido, las sociedades cooperativas fueron utilizadas "como formas de defensa y reacción de amplios sectores de la población mundial que, frente a la insatisfacción de una diversidad de necesidades socioeconómicas, y conscientes de su debilidad individual, han decidido articular sus esfuerzos y fortalecerse a través de la ayuda mutua y la acción común» (Miranda 2012, 26).

Ocurre, sin embargo, que los diferentes cambios socio-económicos que se operan sistemáticamente en la órbita mundial, determinan que las diferentes ramas del Cooperativismo ajusten su quehacer a las demandas y exigencias del mercado.

Por esto, todavía hoy, en el apogeo del siglo XXI, es importante comprender que «el verdadero éxito de la Cooperación y su difusión en el mundo, el paso del tiempo y la inevitable tendencia de las instituciones de asegurar su propia supervivencia aceptando las condiciones del medio, conspiran para aumentar el riesgo de que sus principios sean mal interpretados, diluidos o aún desnaturalizados» (Watkins 1989, 22).

De esta manera, y para preservarse la propia identidad del Cooperativismo, es necesario actualizarse el debate acerca de los principios cooperativos, para que permanezcan revelando la esencia soberana de la cooperatividad como una herramienta socio-económica, sostenida por una orientación moral de solidaridad.

En este sentido, el presente trabajo intenta revelar que ciertas previsiones estatutarias arbitrarias, promovidas por ciertas cooperativas de 
médicos brasileñas, oprimen el efectivo cumplimiento del principio de la adhesión libre o voluntaria: el principio de puertas abiertas.

De otro modo, y considerando el estadio del Derecho, donde se sobrelleva el escenario pos positivista, se quiere enmarcar que cualquier acto que corrompa un principio cooperativo, cualquiera que sea, colisiona con la atmosfera principiológica donde se encuentra inmersa la interpretación y la aplicación del propio derecho.

\section{Una cuestión de principio: ¿pero que es principio?}

En el escenario del Cooperativismo, los principios cooperativos enunciados por la Alianza Cooperativa Internacional $(\mathrm{ACl})$, que también tiene el encargo de revisarlos sistemáticamente, constituyen patrones que orientan el ejercicio de la cooperatividad, delimitando la naturaleza personal, democrática y solidaria de las sociedades cooperativas y de sus miembros.

Este entendimiento, lejos de mostrarse como resultado de una percepción subjetiva, procede de la propia definición de sociedad cooperativa, reconocidas por la $\mathrm{ACl}$ como «una asociación autónoma de personas que se han unido de forma voluntaria para satisfacer sus necesidades y aspiraciones económicas, sociales y culturales en común mediante una empresa de propiedad conjunta y gestión democrática» (ACl 1996, p.73).

Por este camino, y considerada la transcendencia de los principios cooperativos en el ejercicio de la sociedad cooperativa, y de la propia actuación de los socios, es indispensable decirse que el termino principio revela «um padrão que deve ser observado, não porque vá promover ou assegurar uma situação econômica, política ou social considerada desejável, mas porque é uma exigência de justiça ou equidade ou alguma outra dimensão de moralidade» (Dworkin 2002, 36).

Partiendo de su expresión etimológica, es correcto afirmarse que «los principios son mandatos de optimización porque ordenan que algo se realice en la mayor medida posible, de acuerdo con las posibilidades fácticas o jurídicas. Este mandato tiene en realidad un carácter definitivo» (Alexy 2003, 55).

Es cierto que los principios, por su propia definición, son mandamientos nucleares de un determinado sistema, puesto que irradian elementos de conformación de los preceptos normativos o actitudinales, resguardando su esencia o su propio espíritu. En este sentido, no se puede olvidar que: 
Los principios, en tanto objetos de la ponderación, no son entonces mandatos de optimización, sino mandatos que se optimizan o mandatos a optimizar. Como tales, contienen un deber ser ideal, todavía no contienen un deber ser relativo a las posibilidades fácticas y jurídicas. Sin embargo, tiene todo el sentido referirse a los principios como mandatos de optimización. Esta manera de hablar expresa, de la manera más simple, de qué se trata cuando se alude a los principios. De este modo se expresa todo aquello que tiene que ver con los principios y que resulta significativo desde el punto de vista práctico. Este aspecto práctico puede sostenerse además en una reflexión teórica. Existe una relación necesaria entre el deber ser ideal, es decir, entre el principio como tal, y el mandato de optimización, en cuanto regla. El deber ser ideal implica al mandato de optimización y viceversa. Son las dos caras de una misma moneda. (Alexy 2003, 56)

Los principios son directrices basilares. Representan columnas de sustentación de sistemas sociales, económicos, políticos, jurídicos o cooperativos. Simbolizan una fuerza de orientación específica imprescindible que se difunde sobre diferentes preceptos, componiéndoles su pujanza axiológica y sirviendo de criterio para su comprensión e inteligencia (Mello 2000, 48).

\section{Los principios del Cooperativismo}

Hoy por hoy, nadie más duda que por medio de «las cooperativas, el Cooperativismo presenta una estructura organizada para realizar construcciones que, además de alcanzar los campos económicos y sociales, se encarga también del lado moral del individuo, mediante su formación humana» (Miranda 2016, 98).

La comprensión de este enunciado es posible cuando se observa que la esencia de la moral cooperativa estuvo delineada a través de los principios enaltecidos por los miembros de la Sociedad Cooperativa de los Probos de Pioneros de Rochdale. Por esto, es necesario decir que:

A pesar de haber nacido como una cooperativa de consumo, la trascendencia y la repercusión social y económica alcanzada por la Sociedad de los Probos Pioneros de Rochdale es tal que constituye la base de toda clase de cooperativas, incluso las de producción y las de crédito.

En la medida que el triunfo del método cooperacionista de consumo de Rochdale fue progresivamente conocido por distintos lugares de Europa y del mundo, muchas fueron las sociedades constituidas con base en el condicional colaborador y principiológico de los 
Pioneros. Quizás por esto hoy por hoy existe una posición doctrinal unánime que defiende que la Rochdale Society of Equitables Pioneers no ha sido sólo una elaboración práctica en la que él éxito se ha debido a las notables cualidades de los realizadores, sino que desde su nacimiento ha determinado un programa completo que contenía los principios teóricos y las reglas prácticas de organización y funcionamiento de las entidades cooperativas (Miranda 2016, 67).

De una manera muy precisa, «los principios cooperativos que se conocen hoy día no aparecen en los estatutos rochdaleanos a través de un elenco ostensible, pero su presencia es revelada por las normas que han sido establecidas a lo largo del estatuto» (Miranda 2016, 73).

Por esto, la $\mathrm{ACl}$, desde su constitución, se encargó de conformar el estudio, la actualización y el enunciado de los principios cooperativos, puesto que se revisten del aspecto de «verdades universales para la formación y el ensanchamiento de un orden social donde la sociedad libre anhele vivir pacífica y prospera en colaboración. Resisten la ordenación constringente, hacen superfluo todo conflicto destructivo y descentralizan la fiscalización social y económica» (Miranda 2016, 86).

Concisamente, es posible decir que «los Probos de Rochdale han desarrollado una síntesis original de pauta de actuación que ha quedado codificada a través de sus estatutos, que ha constituido el origen de los principios que desde entonces dominan el movimiento cooperativo de todo el mundo» (Miranda 2016, 867). A partir de allí, la ACl esclarece que:

Mucha gente entiende los principios como mandamientos firmes que deben ser seguidos literalmente. En un sentido es verdad, en la medida que deberían proporcionar normas de conducta. En otro sentido, deberían restringir, incluso prohibir, ciertas acciones mientras estimulan otras.

Sin embargo, los principios son más que mandamientos, son pautas para evaluar el comportamiento y tomar decisiones. No es suficiente preguntar si una cooperativa está siguiendo al pie de la letra un principio dado; es igualmente importante saber si está siguiendo el espíritu —si la visión que proporciona cada principio, individual y colectivamente, está arraigada en las actividades diarias de la cooperativa- En este sentido, los principios no son una lista caduca a revisar periódicamente y de forma ritual; son marcos de actuación capacitadores - agentes que dan energía - por los cuales las cooperativas pueden alcanzar el futuro. (ACl 1996, 88).

Los principios constituyen el alma de las sociedades cooperativas, sirven para la puesta en práctica de los valores cooperativos, y no pueden ser observados de manera independiente uno del otro. Hay 
una cohesión dialéctica entre los principios cooperativos que inhibe su aplicación seccionada, fraccionada. El menosprecio de uno, desestima la esencia y efectividad de los demás, corrompiendo la naturaleza de la sociedad cooperativa.

Es, pues, de la aplicación práctica de los principios cooperativos que se tiene garantizada «la naturaleza democrática de las cooperativas, el papel de los diferentes partícipes y la forma de distribuir excedentes» (Gadea, Sacristán y Vasserot 2009, 38).

Los principios cooperativos determinan las cualidades esenciales que hacen que las cooperativas sean diferentes a los otros tipos de empresa y que el movimiento cooperativo sea valioso. Por tanto, su importancia dogmática es evidente, aunque su transcendencia jurídica queda supeditada a los términos en que hayan sido incorporados en las respectivas legislaciones internas, lo que condiciona notablemente su posible eficacia como criterios correctores de posibles impurezas del legislador correspondiente. Ello, no obstante, su carácter informador de la estructura y funcionamiento de la cooperativa, les adiciona un claro valor como elemento de interpretación de la normativa cooperativa (Gadea, Sacristán y Vasserot 2009, 38).

La $\mathrm{ACl}$, a través de la Declaración sobre la Identidad Cooperativa proclamada en el Congreso de Manchester, en el 1995, listó siete principios que no pueden ser despreciados por las cooperativas, y por sus miembros: Adhesión Voluntaria y Abierta; Gestión Democrática por parte de los socios; Participación Económica de los socios; Autonomía e Independencia; Educación, Formación e Información; Cooperación entre Cooperativas; Interés por la Comunidad (ACI 1996).

\section{El principio de la adhesión voluntaria: las puertas abiertas}

Mirando hacia el pasado, se pude verificar que los Probos Pioneros de Rochdale reconocieron el libre acceso de los asociados a través de la regla establecida por el artículo 13 del estatuto sancionado en 1844 y reformado en 1845 (Miranda 2016, 78).

Del análisis de esta regla estatutaria determinante para el ingreso de asociados, es posible concluir que el principio de la adhesión libre no está restringido a la necesidad de confianza mutua. Los aspirantes a ser miembros precisan de, al menos, dos socios que representen su propuesta y les apoyen ante la cooperativa. El ingreso y el alcance de 
la condición de socio están condicionados a la aprobación de la mayoría, así como al pago de una cuota de admisión que podrá ser satisfecha a través de entregas semanales.

Sin embargo, esta normativa puede hallarse en el hecho de que los propios Probos Pioneros de Rochdale, buscando preservar la armonía en el interior de la cooperativa, intentaban admitir como nuevos socios a personas que teniendo capacidades para llegar a ser óptimos cooperativistas, gozasen de su total e incondicional confianza. Esto, por cierto, implicaba que ellos mismos, como responsables del apoyo y presentación del candidato a socio, evidenciasen, de un lado, la reunión de dichas capacidades, y de otro, la oportuna idoneidad de su ingreso (Miranda 2016, 79).

El principio de adhesión voluntaria y abierta determina la necesidad de establecimiento de una dependencia específica entre las cooperativas y las personas a la que sirve substancialmente. Esa dependencia proviene de una relación que "debería definir los negocios realizados por la cooperativa, afectar la forma en la que los hace, y conformar sus planes para el futuro. Además, un reconocimiento de la centralidad de la «adhesión» debe significar particularmente que las cooperativas están comprometidas con un alto nivel de servicios a los socios, la razón principal de su existencia» (ACI 1996, 91).

Por ello, en la descripción del primer principio del Cooperativismo, la $\mathrm{ACl}$ es definitiva en orientar que "las cooperativas son organizaciones voluntarias, abiertas a todas las personas capaces de utilizar sus servicios y dispuestas aceptar las responsabilidades de ser socio, sin discriminación social, política, religiosa, racial o de sexo» (ACI 1996, 74).

No obstante, por la expresión de la Declaración sobre la Identidad Cooperativa, emitida por la $\mathrm{ACl}$, el principio de adhesión voluntaria y abierta es reconocido como el más poderoso, y, de la misma manera, el más infravalorado de todos los principios cooperativos.

Es un principio poderoso porque deja patente que el Cooperativismo no es un sistema discriminatorio, antojadizo, y que las sociedades cooperativas, independientemente de sus ramas, de sus respectivas áreas $u$ objeto de actuación, son accesibles a todas las personas que se muestren dispuestas a participar de su estructura, sirviéndose de los servicios prestados, cumpliendo detalladamente con los preceptos estatutarios y sobrellevando los valores de la cooperatividad, como forma de mantenerse vivo el espíritu cooperativo.

Sin embargo, la adhesión abierta y voluntaria es infravalorada porque aún existen países del mundo en cuyos sistemas legislativos a menudo empujan a la gente hacerse socias de algunas sociedades cooperativas (ACl 1996). Asimismo, en otros, y a despecho de la vigencia y 
especificidad de la legislación cooperativa específica, los gestores de algunos tipos de sociedades cooperativas impiden el acceso libre y voluntario de las personas, creando, o interpretando a su albedrio las pautas de los estatutos, de manera que las mismas sean utilizadas en beneficio de una pequeña parcela de asociados, corrompiendo, de este modo, la naturaleza de la sociedad cooperativa, y soterrando el verdadero sentido del Cooperativismo-cooperativo.

\section{Las puertas abiertas a medias: una práctica arbitraria que impide el cumplimiento del primer principio cooperativo por sociedad cooperativa de médicos brasileña}

En Brasil, el primer principio del Cooperativismo, el principio de adhesión voluntaria y abierta, está reglado por el artículo $4 .^{\circ}$, I, y el artículo 29, y su párrafo primero, de la Ley General del Cooperativismo, Ley 5.764/1971, que disponen:

Art. $4 .{ }^{\circ}$ As cooperativas são sociedades de pessoas, com forma e natureza jurídica próprias, de natureza civil, não sujeitas a falência, constituídas para prestar serviços aos associados, distinguindo-se das demais sociedades pelas seguintes características:

I - adesão voluntária, com número ilimitado de associados, salvo impossibilidade técnica de prestação de serviços

Art. 29. O ingresso nas cooperativas é livre a todos que desejarem utilizar os serviços prestados pela sociedade, desde que adiram aos propósitos sociais e preencham as condições estabelecidas no estatuto, ressalvado o disposto no artigo $4 .^{\circ}$, item I, desta Lei.

$\S 1 .^{\circ}$ A admissão dos associados poderá ser restrita, a critério do órgão normativo respectivo, às pessoas que exerçam determinada atividade ou profissão, ou estejam vinculadas a determinada entidade (Miranda, Galhardo y Gonçalves 2013, 64 y 71)

De acuerdo con el razonamiento de la incidencia de los principios al marco legislativo de un determinado sistema jurídico, la Ley brasileña adopto la adhesión voluntaria y abierta como vértice de ordenación de las normas estatutarias de las diferentes cooperativas de Brasil. Es decir, ninguna especie de cooperativa puede contrariar la norma legal, y dejar de atender el mandamiento de la $\mathrm{ACl}$, en el sentido de oprimir el pleno acceso de la gente a la cooperativa.

A partir de una interpretación del propio concepto de cooperativa, formulado por la $\mathrm{ACl}$, es posible entender que las cooperativas, de cualquier rama, están abiertas a todas las personas «capaces de utilizar sus 
servicios y dispuestas a aceptar las responsabilidades de ser socio, sin discriminación social, política, religiosa, racial o de sexo» (ACI 1996, 89).

A lo largo de la Declaración sobre la Identidad Cooperativa, la ACI «reafirma un compromiso general básico para las cooperativas, desde su surgimiento en el siglo XIX: un compromiso con el reconocimiento de la dignidad fundamental de todos los individuos, de hecho, de todas las personas» (ACl 1996, 89). Es decir, el Cooperativismo, y las cooperativas, son para todos, están siempre accesibles a quienes les apetecieren hacer parte de una cooperativa, siempre que se ajusten a los preceptos normativos elaborados en constancia a los valores y principios cooperativos.

En líneas generales, se puede comprender que la $\mathrm{ACl}$ no quiere que las cooperativas promuevan acciones que imposibiliten el acceso a condición de socio por cualquier persona que se muestre apta al cumplimiento de las obligaciones que tendrá para con la cooperativa, "obligaciones que varían de cooperativa en cooperativa, pero incluyen el ejercicio del derecho de voto, la participación en las reuniones, la utilización de los servicios de la cooperativa y la aportación de capital cuando sea necesaria» (ACI 1996, 89).

Pese a esta determinación inherente al principio de puertas abiertas, nítidamente correlacionada con los valores de la democracia, igualdad, solidaridad y equidad, existen cooperativas de médicos brasileñas que redimensionan las directrices de la legislación, y el espíritu de los valores y principios, instituyendo clausulas estatutarias abiertas, de ámbito general e interpretación difusa, que permite a los gestores inhibir la libre adhesión, a penas para justificar los intereses o la inconveniencia de aquellos que ya se encuentran en el cuadro de miembros.

Ejemplo de cláusulas que inhiben la libre adhesión son que aquellas impeditivas de ingreso por imposibilidad técnica, que veta el ingreso de nuevo miembro que no se muestre apto técnicamente a la «prestación de los servicios al asociado por la cooperativa, para el cumplimiento de su objeto social».

Ocurre que el impedimento de acceso al cuadro de miembros por imposibilidad técnica es una norma opresora, de aspecto arbitrario, que afecta la legalidad de los estatutos, una vez que sus cláusulas determinan que compite al Consejo de Administración la definición acerca del sentido del impedimento emanado de la dicha imposibilidad técnica. Esto, es cierto, contamina el sentido normativo de los estatutos sociales de un acentuado aspecto de subjetividad, teniendo en cuenta que será un grupo reducido de socios, los que integran el Consejo de Administración, que decidirán sobre el ingreso de un determinado candidato a miembro, en virtud de la imposibilidad técnica. 
De la misma forma, en Brasil, existen cooperativas de médicos que utilizan otros artificios para frenar el libre acceso de nuevos asociados, mientras establecen que la aprobación del ingreso de candidato esta subrogada a aprobación en proceso selectivo, o al cumplimiento de un curso previo de formación cooperativa.

Dichas cooperativas llaman, a esta interpelación, de principio de integración, por lo cual determinan que toda persona que desee participar del cuadro asociativo participe de ponencias, cursos o cualquier otra forma de capacitación cooperativa, que deberá ser ofrecida en un plazo no superior a sesenta días contados de la aprobación del ingreso del candidato.

Estas exigencias, contrarias a los elementos que totalizan el principio de adhesión libre y voluntaria, pero utilizadas constantemente para frenar la integración de nuevos miembros a distintas cooperativas de médicos en Brasil, provoca la judicialización de las respectivas cuestiones, puesto que los candidatos perjudicados someten el veto de adhesión a la Tutela del Estado, a través de procesos judiciales largos, exhaustivos, que exponen el Cooperativismo al sistema jurídico brasileño, cuyos jueces critican la ruptura de la identidad cooperativa y el rompimiento de la propia génesis de la cooperación. Sobre ello, es posible ver la posición del Superior Tribunal de Justicia brasileño, a través de la descripción siguiente:

Relator: Ministro Ricardo Villas Bôas Cueva agravante: Unimed Campinas Cooperativa de Trabalho Médico advogado: Dagoberto Silverio Da Silva e outro(s) agravado: Bruno Franceschetti Santa Rosa

Advogados: Fábio Gindler De Oliveira e outro(s) Paulo Augusto Rolim De Moura e outro(s) ementa

Agravo regimental nos embargos de declaração no agravo em recurso especial. Civil. Cooperativa de trabalho médico (UNIMED). Ingresso de novo associado. Recusa. Exigência de aprovação em processo seletivo e realização de curso. Incidência do princípio da porta aberta (livre adesão). 1. O ingresso nas cooperativas é livre a todos que desejarem utilizar os serviços prestados pela sociedade sendo, em regra, ilimitado o número de associados, salvo impossibilidade técnica de prestação de serviços (arts. $4 .{ }^{\circ}$, I, e 29 da Lei n. ${ }^{\circ}$ 5.764/1971). Incidência do princípio da livre adesão voluntária. 2. Pelo princípio da porta-aberta, consectário do princípio da livre adesão, não podem existir restrições arbitrárias e discriminatórias à livre entrada de novo membro na cooperativa, devendo a regra limitativa da impossibilidade técnica de prestação de serviços ser interpretada segundo a natureza da sociedade cooperativa. 3. Agravo regimental não provido. (Superior Tribunal de Justicia - AgRg nos EDcl no AGRAVO EM RECURSO ESPECIAL N. ${ }^{\circ} 667.072$ - SP (2015/0041255-6), disponible en 
«https://ww2.stj.jus.br/processo/revista/documento/mediado/?compo nente $=\mid$ TA\&sequencial $=1492460 \&$ num_registro $=201500412556 \&$ da $\mathrm{ta}=20160310 \&$ formato=PDF»)

Indiferentes a la distinción de la cooperativa en relación a los demás modelos de empresas reconocidas por el Derecho, las prácticas utilizadas por algunas cooperativas, a través de la promoción de medios impeditivos al ingreso de nuevos miembros al cuadro asociativo, rompen la particularidad del principio de adhesión libre y voluntaria.

Tales actitudes dejan claro que en estas entidades, conducidas por personas específicas sin una huella cooperativa, tanto persiguen intereses muy específicos, contrarios al espíritu del Cooperativismo, como preservan las puertas de la cooperativa abiertas a medias, una vez que el acceso de nuevo socio pierde la impersonalidad, deja de ser libre, y suele ser permitido para aquellos que se ajustan a la voluntad y al gusto de los directivos.

Esto, no se puede negar, corrompe la tenacidad axio-principiológica de la cooperatividad.

\section{A título de conclusión}

Es indudable que el principio de la adhesión libre y voluntaria está «estrechamente unido con el principio de la educación y el principio de la gestión democrática por parte de los socios. Los socios solamente pueden desempeñar su papel si están informados y si hay comunicación eficaz entre los socios, líderes elegidos, directivos y empleados (si los hay)» (ACl 1996, 91).

Por esto, debe prevalecer en el orden cooperativo mundial, y ser acepto por todas las cooperativas, mismo las sociedades cooperativas de médicos, la máxima orientación de que toda entidad que nace bajo el perfil de la cooperatividad está abierta a todos los individuos que deseen tornarse cooperativistas, integrando, así, el cuadro asociativo de la cooperativa con la cual tiene identidad funcional.

De esta manera, las exigencias definidas para el ingreso de un nuevo miembro al cuadro asociativo deben ser coherentes con el objeto social, reconociendo las condiciones de honor y moral del candidato, y las circunstancias que viabilicen su devoción a los valores y principios del Cooperativismo.

Por supuesto, ninguna cooperativa podrá utilizarse, arbitrariamente, de exacciones de calificación o formación cooperativa como criterio de admisión de candidato, pues la exigencia de la formación e in- 
formación cooperativa se muestra posible solamente después que se confirme la condición de miembro.

Asimismo, en relación a las posibilidades técnicas de prestación de servicio al candidato a miembro no puede ser adoptada como herramienta de reserva de mercado para la actuación de aquellas personas que ya se encuentra socios de la cooperativa. El espacio técnico-profesional no puede ser garantizado a través de la fuerza de la cooperativa, sino que por los propios atributos profesionales y personales del médico.

El principio de adhesión libre y voluntaria no puede ser despreciado por las cooperativas que pretendan acceder su ejercicio a la génesis identitaria del Cooperativismo, pues su descrédito lesiona la identidad cooperativa y afecta la credibilidad del sistema, que encuentra amparo sobre una columna de aspecto moral.

Hay que enmarcarse que el sentido de mantenerse la cooperativa dentro de una perspectiva de puertas abiertas existe desde el surgimiento de la Sociedad Cooperativa de los Probos Pioneros de Rochdale, cuando sus fundadores previeron ya la posibilidad de nuevas personas adherir al estatuto social, robusteciendo la base personal de la sociedad al objeto de hacerlas partícipes de los resultados de la actividad económico-social por ella desarrollada (Miranda 2016).

De este modo, y en el momento que el mundo enfrenta crisis de diversos talantes, y Brasil se encuentra ahogado en una crisis de valor, de ética, y de derrumbamiento de la fortaleza de las instituciones que debería ofrecer sostenibilidad al Estado Democrático de derecho, es importante rescatarse la máxima de que un principio traduce una norma que impone la condición del deber-ser, en este caso, el deber ser co-operativo, y no selectivo.

El Cooperativismo, siendo un sistema uno, que orienta y abriga cooperativas que desarrollan actividades congéneres con una dialéctica ético-moral, no puede permitir que los cooperativistas, y sus respectivas cooperativas, olviden que los principios son, "dotados de cogência e imperatividade, não podem ser relegados aos casuísmos de quem quer que seja, posto que já são a própria essência e substância da consciência jurídica em determinado seio coletivo» (Leite e Leite, 2003, 143).

No siendo esta la directriz de las cooperativas, el Cooperativismo se encontrará ante el riesgo de perder definitivamente su razón de ser, y ver la decadencia de su de representatividad como el sistema que posibilita el desarrollo socioeconómico del hombre, y le permite una evolución moral y espiritual (Miranda 2008, 316). 


\section{Referencias}

ALIANZA COOPERATIVA INTERNACIONAL. 1996. «Declaración de la Alianza Cooperativa Internacional sobre la Identidad Cooperativa aprobada en Manchester.» Anuario de Estudios Cooperativo. Bilbao: Universidad de Deusto.

ALEXY, Robert. 2003. "Tres escritos sobre los derechos fundamentales y la teoría de los principios». N 28. Serie de teoría jurídica y filosofía del Direito. Universidad de Externado de Colombia: Bogotá.

ARIZMENDIARRIETA, José Maria. 1983. Pensamientos. Caja laboral Popular: Estella.

DIVAR, Javier. 1985. La alternativa cooperativa: una respuesta ante la crisis. Barcelona: Ceac.

DWORKIN, Ronald. 2002. Levando os Direitos a sério. Tradução de Nelson Boeira. São Paulo: Martins Fontes.

ESPÍNDOLA, Ruy Samuel. 2002. Conceito de princípios constitucionais. 2 ed. São Paulo: RT.

GADEA, Enrique; SACRISTÁN, Fernando y VASSEROT, Carlos Vargas. 2009. Régimen jurídico de la sociedad cooperativa del siglo XXI. Realidad actual y propuestas de reforma. Madrid: Dykinson, S.L.

LEITE, George Salomão e LEITE, Glauco Salomão. 2003. «A abertura da constituição em face dos princípios» in LEITE, George Salomão. Organizador. Dos princípios constitucionais. Considerações em torno das normas principiológicas da constituição. São Paulo: Malheiros.

MEINEN, Ênio y GAUDIO, Ronaldo. 2015. "Sobre o diferencial estructural e desafíos das instituiçoes financieras cooperativas no ambiente regulatório brasileiro». Boletín de la Asociación Internacional de Derecho Cooperativo 49: 137-180.

MELLO, Celso Antônio Bandeira de. 2000. Curso de direito administrativo. 12. ed. São Paulo: Malheiros Editores.

MIRANDA, José Eduardo. 2008. «De la cooperación al cooperativismo: análisis de los valores y principios cooperativos como instrumentos de regeneración de entornos debilitados.» Tesis doctoral. Universidad de Deusto.

MIRANDA, José Eduardo de, GALHARDO, José Henrique da Silva e VIEIRA, Paulo Gonçalves. 2013. Regime jurídico da sociedade cooperativa. Curitiba: Juruá.

MIRANDA, José Eduardo de. 2012. De la crisis de identidad al rescate de la génesis del Cooperativismo. Madrid: Dykinson.

MIRANDA, José Eduardo. 2014. «De la propedéutica de los principios cooperativos a la intercooperación como pilastra del cooperativismo». Boletín de la Asociación Internacional de Derecho Cooperativo 48: 149-164.

MIRANDA, José Eduardo. 2015. «El sistema de crédito cooperativo brasileño y la identidad cooperativa: la necesidad de vigilancia permanente de los valores del cooperativismo para la sostenibilidad del modelo». Boletín de la Asociación Internacional de Derecho Cooperativo 49: 65-82. 
MIRANDA, José Eduardo. 2016. Filosofía cooperativa: análisis del proceso de conformación del Cooperativismo. Lisboa: Juruá.

Superior Tribunal de Justicia - AgRg nos EDcl no AGRAVO EM RECURSO ESPECIAL N. ${ }^{\circ} 667.072$ - SP (2015/0041255-6), disponible en «https://ww2.stj. jus.br/processo/revista/documento/mediado/?componente=ITA\&sequenci $\mathrm{al}=1492460 \&$ num_registro $=201500412556 \&$ data $=20160310 \&$ formato $=$ PDF»

WATKINS, W. P. 1989. Los principios cooperativos hoy y mañana. Esacoop: Bogotá. 


\section{Derechos de autor}

El Boletín de la Asociación Internacional de Derecho Cooperativo es una revista de acceso abierto lo que significa que es de libre acceso en su integridad inmediatamente después de la publicación de cada número. Se permite su lectura, la búsqueda, descarga, distribución y reutilización legal en cualquier tipo de soporte sólo para fines no comerciales y según lo previsto por la ley; sin la previa autorización de la Editorial (Universidad de Deusto) o el autor, siempre que la obra original sea debidamente citada (número, año, páginas y DOI si procede) y cualquier cambio en el original esté claramente indicado.

\section{Copyright}

The International Association of Cooperative Law Journal is an Open Access journal which means that it is free for full and immediate access, reading, search, download, distribution, and lawful reuse in any medium only for non-commercial purposes, without prior permission from the Publisher or the author; provided the original work is properly cited and any changes to the original are clearly indicated. 